\title{
„Das Barbie Syndrom“. Ein Fallbericht über die Körperdysmorphe Störung
}

\author{
Maria Gruber $\cdot$ Rebecca Jahn $\cdot$ Karin Stolba $\cdot$ Michael Ossege
}

Eingegangen: 2. Mai 2017 / Angenommen: 9. Juli 2017 / Online publiziert: 8. August 2017

(c) Der/die Autor(en) 2017. Dieser Artikel ist eine Open-Access-Publikation.

\section{Zusammenfassung}

Hintergrund Der vorliegende Fallbericht beschreibt eine 37-jährige Patientin, die ihre Faszination für „Barbie-Puppen“von der Kindheit bis ins Erwachsenenalter beibehalten hat und entsprechende Abweichungen davon an ihrem Körper schlecht tolerieren konnte. Die stationäre Aufnahme an der psychosomatischen Station erfolgte auf Grund einer Essstörung.

Methodik Die Diagnostik wurde mittels ICD-10 und DSM-5 Kriterien für Achse I Störungen und mittels SKID II Interview (deutsche Version für DSM-4) für Achse II Störungen durchgeführt. Zur störungsspezifischen Diagnostik wurde die „Modifizierte Version der Yale-Brown Obsessive Compulsive Scale für Körperdysmorphe Störung" angewendet.

Ergebnisse Bei der Patientin wurde eine Dysmorphophobie (ICD-10: F45.21) oder eine körperdysmorphe Störung (DSM-5: 300.7) sowie eine Bulimia nervosa (ICD-10: F50.2; DSM-5: 307.51) diagnostiziert. Weiters erfüllte sie die Kriterien für eine selbstunsichere, depressive und histrionische Persönlichkeitsstörung. Es erfolgte eine medikamentöse Einstellung auf Fluoxetin und eine psychotherapeutische Arbeit mit dem Körperbild, am Selbstkonzept und den Schamgefühlen.

Schlussfolgerung Anhand des vorliegenden Fallberichtes soll auf die körperdysmorphe Störung als eigene Krankheitsentität mit hoher Prävalenz hingewiesen werden. Unterschiedliche diagnostische Einordnungen und die Komorbidität mit Essstörungen werden diskutiert. Die körperdysmorphe Störung kann in der Praxis, gerade bei Vorliegen einer Essstörung, leicht

Dr. M. Gruber $(\bowtie) \cdot$ R. Jahn $\cdot$ K. Stolba $\cdot$ M. Ossege Abteilung für Sozialpsychiatrie, Universitätsklinik für Psychiatrie und Psychotherapie, Medizinische Universität Wien, Währinger Gürtel 18-20, 1090 Wien, Österreich maria.gruber@meduniwien.ac.at übersehen werden, jedoch ist die Diagnosestellung für das weitere therapeutische Vorgehen relevant.

Schlüsselwörter Dysmorphophobie · Körperdysmorphe Störung · Bulimia nervosa

\section{'Barbie Doll Syndrome'. A case report of body dysmorphic disorder}

\section{Summary}

Background This case report aims to present a 37year-old women striving to shape her body like a Barbie doll of which she has been fascinated since childhood. She could hardly tolerate any deviation from this beauty ideal. She has been admitted to the psychosomatic ward due to an eating disorder.

Methods The ICD-10 and DSM-5 criteria were established for axis I disorders and the German version of the SCID II interview (for DSM-4) was applied for axis II disorders. Additionally, the "modified YaleBrown Obsessive Compulsive Scale for body dysmorphic disorder" was carried out.

Results The diagnosis of dysmorphophobia (ICD-10: F45.21) or body dysmorphic disorder (DSM-5: 300.7) and bulimia nervosa (ICD-10: F50.2; DSM-5: 307.51) was confirmed. The patient fulfilled criteria of an avoidant, depressive and histrionic personality disorder. Psychopharmacological treatment with Fluoxetine was started and the patient participated in an intensive inpatient psychosomatic program. The body image, self-concept and the sense of shame were therapeutic key topics.

Conclusion The present case report focuses on body dysmorphic disorder as a distinctive entity with high prevalence. Diagnostic criteria of different classification systems were contrasted and comorbidity with eating disorders was discussed. In clinical praxis, body dysmorphic disorder remains underdiagnosed, espe- 
cially when cooccurring with an eating disorder. However, the correct diagnosis could be relevant for therapy planning.

Keywords Body dysmorphic disorder · Bulimia nervosa

\section{Einleitung}

Die Unzufriedenheit mit dem eigenen Körper bzw. Körperbild ist bei Mädchen oder Frauen in der Allgemeinbevölkerung weit verbreitet und wird auf Grund der Häufigkeit oft als „normative Einstellung“ interpretiert. Diese Unzufriedenheit steht auch im Zusammenhang mit der Entwicklung einer körperdysmorphen Störung (KDS), welche mit einer erheblichen Beeinträchtigung der Lebensqualität einhergeht. Der Erkrankungsbeginn der KDS liegt typischerweise in der Adoleszenz [1]. Die KDS hat eine Prävalenz von $1,9 \%$ in der Gesamtbevölkerung und kommt bei Patientinnen mit kosmetischen Eingriffen (13,2\%) oder in dermatologischen Behandlungen (11,3\%) deutlich häufiger vor [2]. Der Verlauf der KDS ist normalerweise primär chronisch mit einer mittleren Krankheitsdauer von 16 Jahren, obwohl eine Besserung durch entsprechende Behandlung erzielt werden kann [3, 4]. Die KDS beschreibt eine ständige Beschäftigung mit einem oder mehreren wahrgenommenen körperlichen Makeln, der oder die für andere Personen kaum oder nicht sichtbar ist oder sind. „Die Entstellungsangst" wurde 1886 erstmalig durch den italienischen Arzt und Psychiater Morselli beschrieben [5] und hat zwei Jahrhunderte später nicht an Bedeutung verloren.

\section{Dysmorphophobie oder Körperdysmorphe Stö- rung - Probleme der Differenzialdiagnostik}

Heute wird die Dysmorphophobie (F45.21) in der ICD-10 [6] als somatoforme Störung im Sinne einer Hypochondrie oder als anhaltend wahnhafte Störung (F22.8) klassifiziert. Im DSM-4 wurde die körperdysmorphe Störung noch der somatoformen Störungsgruppe zugeordnet und im DSM-5 im Kapitel „Zwangsstörungen“ kategorisiert. Die Diagnose wird im DSM-5 anhand von mehreren Abstufungen unterteilt: von guter Krankheitseinsicht bis $\mathrm{zu}$ unkorrigierbaren Überzeugungen über die entstellte körperliche Erscheinung (Tab. 1). Eine komorbide wahnhafte Störung mit körperbezogenem Wahn (297.1) sollte nur diagnostiziert werden, wenn andere körperbezogene Überzeugungen vorhanden sind, die im Rahmen des Erkrankungsbildes nicht erklärbar sind (z. B. Dermatozoenwahn) [4].

Anhand der Falldarstellung möchten wir eine Patientin mit einer Dysmorphophobie und einer Bulimia nervosa präsentieren, welche seit vielen Jahren und mit viel Aufwand versucht ihrem plastischen Schönheitsideal „Barbie“ so nah wie möglich zu kommen.

\section{Fallbericht}

Frau B., eine 37-jährige Patientin kommt zur dritten informellen Aufnahme an die Station mit Schwerpunkt Psychosomatik. Vordergründig besteht eine Verschlechterung der bulimischen Symptomatik mit einer Zunahme der Häufigkeit der Ess-/Brechanfälle und eine Angst vor Komplikationen. Zweimalig wäre nach Kollapsen/Synkopen in Notaufnahmen eine Hypokaliämie diagnostiziert und substituiert worden.

Tab. 1 Unterschiedliche diagnostische Einordnung der Körperdysmorphen Störung (KDS) in der ICD-10 als Dysmorphophobie oder wahnhafte Störung bzw. im DSM V als Körperdysmorphe Störung

\begin{tabular}{|c|c|c|}
\hline ICD-10 Dysmophophobie (F45.21) & $\begin{array}{l}\text { ICD-10 Wahnhafte } \\
\text { Störung (F22.8) }\end{array}$ & DSM V Körperdysmorphe Störung bzw. engl. Body Dysmorphic Disorder(300.7) \\
\hline \multicolumn{2}{|c|}{ Beharrliche Beschäftigung mit der körperlichen Erscheinung } & $\begin{array}{l}\text { A) Übermäßige Beschäftigung mit einem oder mehreren wahrgenommenen Mängeln } \\
\text { oder Defekten im äußeren Erscheinungsbild, die für andere nicht erkennbar sind oder } \\
\text { geringfügig erscheinen }\end{array}$ \\
\hline \multicolumn{2}{|c|}{$\begin{array}{l}\text { Normale oder allgemeine Körperwahrnehmungen werden von } \\
\text { der betreffenden Patientin oder dem betroffenen Patienten oft als } \\
\text { abnorm und belastend interpretiert }\end{array}$} & $\begin{array}{l}\text { B) Im Verlauf der Störung hat die Person in Reaktion auf die Befürchtungen bezüglich } \\
\text { des Aussehens sich wiederholende Verhaltensweisen (z. B. Überprüfung im Spiegel, } \\
\text { übermäßige Körperpflege, Hautzupfen/-quetschen, Rückversicherungsverhalten) oder } \\
\text { mentale Handlungen (z. B. Vergleich des Aussehens mit dem von anderen) ausgeführt }\end{array}$ \\
\hline \multicolumn{2}{|c|}{$\begin{array}{l}\text { Die Aufmerksamkeit wird meist nur auf einen oder mehrere wahr- } \\
\text { genommene Makel des Körpers fokussiert }\end{array}$} & $\begin{array}{l}\text { C) Die übermäßige Beschäftigung verursacht in klinisch bedeutsamer Weise Leiden oder } \\
\text { Beeinträchtigungen in sozialen, beruflichen oder anderen wichtigen Funktionsbereichen }\end{array}$ \\
\hline- & $\begin{array}{l}\text { + unkorrigierbare } \\
\text { Gewissheit über die } \\
\text { körperliche Entstellung }\end{array}$ & $\begin{array}{l}\text { D) Die übermäßige Beschäfttigung mit dem äußeren Erscheinungsbild kann nicht besser } \\
\text { durch Befürchtungen in Bezug auf Körperfett oder -gewicht erklärt werden, wie sie bei } \\
\text { Personen auftreten, deren Symptomatik die diagnostischen Kriterien für eine Essstörung } \\
\text { erfüllt }\end{array}$ \\
\hline- & - & $\begin{array}{l}\text { Bestimmung: } \\
\text { Gute Krankheitseinsicht bis nicht korrigierbare Gewissheit über die Körperliche Entstel- } \\
\text { lung }\end{array}$ \\
\hline \multicolumn{2}{|c|}{$\begin{array}{l}\text { Im ICD-10 kann entweder eine Dysmorphophobie (F45.21) oder } \\
\text { eine sonstige anhaltend wahnhafte Störung (F22.8) diagnostiziert } \\
\text { werden }\end{array}$} & $\begin{array}{l}\text { Im DSM V wird die körperdysmorphe Störung mit oder ohne wahnhaften Überzeugun- } \\
\text { gen diagnostiziert }\end{array}$ \\
\hline
\end{tabular}


Seither würde die Patientin Palpitationen verspüren und hätte Angst vor Herzrhythmusstörungen. Die Patientin ist untergewichtig mit einem Körpergewicht von $43 \mathrm{~kg}$ bei einer Körpergröße von $155 \mathrm{~cm}$ (BMI $17,9 \mathrm{~kg} / \mathrm{m}^{2}$ ).

Das Erscheinungsbild der Patientin ist eindrücklich. Ihr artifizieller Stil mit toupierten Haaren und künstlichen Haarteilen (Beehive-Frisur), ein stark geschminktes Gesicht mit Betonung der Augen umrahmt durch den dicken Lidstrich mit den angebrachten falschen Wimpern und durch die zarte, etwas vorgealterte Haut mit dem dunklen solariengebräunten Teint stechen ins Auge. Der Körperbau ist zart mit Ausnahme der großen Brüste.

\section{Psychosoziale Entwicklung}

Die Patientin ist mit einer eineiigen Zwillingsschwester und einer älteren Schwester in stabilen familiären Verhältnissen aufgewachsen. Sie beschreibt die Zwillingsschwester als ihr „Spiegelbild“, beide hätten schon immer viel Wert auf ihr Aussehen gelegt, sich miteinander verglichen und sich gegenseitig korrigiert und kritisiert. In der Schule wären die Schwestern auch manchmal auf Grund ihrer „Pausbäckchen“ gehänselt worden. Weiters beschreibt sie eine große Faszination für Barbiepuppen, die bis heute anhalten würde. Mit 17 Jahren lernte sie ihren Ex-Mann kennen, mit dem sie sich verlobte und zwei Kinder bekam. Nach der Geburt des zweiten Kindes kam es zu einer postpartalen Depression mit einer daraus resultierenden Bindungsstörung zum Sohn. Die Patientin suchte keine professionelle Hilfe auf. Vier Jahre später beschreibt sie eine zweijährige „glückliche Phase“, welche sie auf das erfolgreiche Berufsleben (guter Verdienst als ungelernte Hilfskraft) zurückführt. Sie hätte Anerkennung erhalten und sich mehr Selbstachtung zugestehen können.

Gleichzeitig ließ sie eine Brustvergrößerung bei einer zuvor normal-proportionierten Brust und eine Fettabsaugung der Fettpölster im Bereich der Knie machen. Die kosmetischen Operationen wurden in einer Klinik in Tschechien vorgenommen. Auf Grund von Komplikationen durch eine einseitige Kapselfibrose an der Brust habe die Patientin anhaltende Schmerzen und es erfolgten weitere Besuche bei einem plastischen Chirurgen.

Als die Patientin die Arbeitsstelle verlor (nach dem Konkurs der Firma), reagierte sie mit sozialem Rückzug und depressiver Symptomatik. Eine Gewichtszunahme von mehreren Kilos auf $53 \mathrm{~kg}$ folgte, die Patientin war aber weiterhin normalgewichtig. Ein Kommentar einer Bekannten, die sich erkundigte, ob die Patientin erneut schwanger wäre, hätte eine Krise ausgelöst. Die Patientin begann im Alter von 27 Jahren mit selbst-induziertem Erbrechen zur Gewichtsregulation und entwickelte das Vollbild einer Bulimia nervosa. Die Beziehung zum Lebensgefährten scheiterte, Frau B. bezieht aktuell Notstandshilfe und ist ge- ringfügig im Verkauf tätig. Die Patientin lebt seit der Trennung vom Ex-Lebensgefährten wieder bei ihren Eltern. Im 5 Personen Haushalt leben die Patientin, ihre zwei Kinder (Sohn und Tochter) und ihre Eltern.

\section{Frühere Behandlungen}

Die Patientin begab sich drei Jahre nach Beginn der Essstörung in ambulante Behandlung (Einzel und Gruppentherapie) bei einer auf Essstörungen spezialisierte Einrichtung. Zwei Jahre später erfolgte der erste stationäre Aufenthalt im Rahmen des 8-wöchigen psychotherapeutischen Turnus auf der Station 06B. Danach gelang es ihr für 3 Monate die Ess-/ Brechanfälle zu unterlassen. Ein halbes Jahr später erfolgte ein weiterer Turnus. Danach konnte die Symptomatik jedoch nur in den Hintergrund gedrängt werden. Die Patientin nahm berufliche Veränderungen vor und begann eine Lehre als pharmazeutischkaufmännische Assistentin, welche sie jedoch nicht abschloss. Eine zweijährige Psychotherapie wurde auf Grund von unzureichendem Fortschritt beendet und eine psychopharmakologische Behandlung wurde bisher aus Angst vor Unverträglichkeiten abgelehnt.

\section{Psychopathologischer Status bei Aufnahme}

Die Patientin leidet an schwer korrigierbaren Überzeugungen über die körperliche Entstellung, einer Störung der Körperwahrnehmung (,der Bauch wäre zu dick“, „die Beine wären zu gerade“, „der Hintern wäre zu flach“, „die Cellulite wäre zu stark“, „die Haut zu blass“ u.v.m.) und Zwangsgedanken durch den ständigen Vergleich des eigenen Aussehens mit anderen. Es bestehen zahlreiche auf den Körper bezogene Ängste („zu dick zu werden“, „die Haare könnten verrutschen“, „die Schminke könnte verwischen“) sowie auch sozialphobische Ängste („Angst auf Grund des Aussehens und Verhaltens auf andere beschämend oder peinlich zu wirken“). Die Patientin beschäftigt sich zwanghaft mit Essen und dem äußeren Erscheinungsbild und investiert bis zu drei Stunden täglich in die Körperpflege und Kosmetik (Camouflage) und zwei bis drei Stunden täglich in den Ablauf ritualisierter Ess-/Brechanfälle. Gleichzeitig kommt es auch zu einer starken Rückversicherungstendenz mit häufigen Blicken in den Spiegel, Selfies oder Rückfragen. Sie zeigt ein Vermeidungsverhalten (unterlässt es vollständig Fremden ungeschminkt vor die Augen $\mathrm{zu}$ treten, öffentliche Bäder aufzusuchen oder sich bei Licht nackt zu zeigen). Die Stimmungslage der Patientin ist subdepressiv und der Affekt verarmt. Frau B. berichtet über übermäßige Schamgefühle und gelegentliche Stimmungsschwankungen (besonders bei den Essattacken würden euphorische Gefühle auftreten, die dann oft mit dysphorischen abwechseln). Die Impulskontrolle wäre bei Heißhungerattacken mit Essanfällen und anschließenden selbstinduziertem Erbrechen gestört. Außerdem beschreibt die Patientin 
eine durchgehende Libidostörung und leichte Einund Durchschlafstörungen. Sie klagt über gelegentlichen Lebensüberdruss, es bestehen keine Suizidgedanken oder -versuche in der Anamnese.

\section{Diagnostik}

Die Patientin erfüllt die ICD-10 Kriterien für eine Dysmorphophobie (F45.21) und eine Bulimia nervosa (F50.2). Es wurde zudem ein störungsspezifisches semistrukturiertes Interview, die „Modifizierte Version der Yale-Brown Obsessive Compulsive Scale für Körperdysmorphe Störung“ (BDD-YBOCS) [7], welches auch 3 diagnostische Kriterien des DSM-5 enthält, angewendet. Das Ergebnis deutet auf eine schwere körperdysmorphe Störung hin (33 von 48 Punkten). Eine SKID II Diagnostik ergab eine Persönlichkeitsstörung mit selbstunsicheren, depressiven und histrionischen Merkmalen. Im klinischen Eindruck imponieren die starke Unsicherheit und Schamgefühle der Patientin auch als Ausdruck eines „verdeckten“ Narzissmus. Bei diesem sind die narzisstischen Merkmale im Verhalten nicht beobachtbar wie bei „offenem“ Narzissmus, sondern hinter einem unsicheren Auftreten verborgen, und gehen mit niedrigem Selbstwert und hoher Bindungsangst einher [8]. Auf die Entwicklung einer KDS bei einer typischerweise bestehenden narzisstischen Verwundbarkeit gepaart mit hypochondrischen Ängsten wurde hingewiesen [9].

\section{Psychotherapeutische Behandlung}

Ein Cochrane Literatur Review liefert drei randomisierte kontrollierte Studien (RCT) zur Wirksamkeit von Psychotherapie bei der KDS. Dabei wurde die kognitive Verhaltenstherapie und Expositionsbehandlungen untersucht, welche Effekte auf die klinische Symptomatik hatten, die auch über das Therapieende hinaus bestanden. In der Behandlung der KDS erwies sich die Kombinationstherapie aus Psycho- und Psychopharmakotherapie am wirkungsvollsten [10].

Im Rahmen der Behandlung an der Station für Psychosomatik erfolgte eine Auseinandersetzung mit den der körperdysmorphen Störung und der Bulimia nervosa zugrundeliegenden Problemen der Patientin in einem Einzel- und Gruppentherapeutischen Setting. Obwohl die Patientin wesentliche Funktionseinschränkungen im Leben hat, konnten Kernthemen in der Therapie besprochen werden. Insbesondere war es nach entsprechender therapeutischer Beziehungsarbeit möglich sich dem zentralen Affekt "Scham“ anzunähern und die vermeintliche Entstellung zu besprechen.

Die Störung zeigte sich bei der Patientin in mehreren Bereichen, das Köperbild, das Selbstkonzept und interpersonale Beziehungen betreffend [11]. Bei der Patientin bestehen Defizite in der Selbstwahrnehmung sowie ein vorherrschendes negatives Selbstbild. Die Entwicklung einer körperdysmorphen Er- krankung geht aus psychodynamischer Perspektive mit einer Entfremdung von sich selbst einher [12]. Weiters bestehen Schwierigkeiten eine befriedigende Partnerschaft einzugehen, der Mutterrolle gerecht zu werden sowie Autonomie-Abhängigkeitskonflikte mit primären Bezugspersonen. Der zentrale Affekt der körperdysmorphen Störung ist die Scham [3, 11]. Auch bei unserer Patientin stellt sie eine zentrale Emotion dar, welche sie daran hindere, sich beispielsweise aktiv an Gruppentherapien zu beteiligen. Die Angst vor einer peinlichen Bewertung durch andere wäre dabei zu groß. Im Rahmen der Gruppentherapien erfolgte unter anderem eine intensive Auseinandersetzung der TeilnehmerInnen mit der Emotion „Scham“. Die Patientin konnte sich in vielen Schilderungen der anderen Patientinnen wiedererkennen, jedoch nur wenig von sich einbringen.

Im stationären Aufenthalt kam es zu einem vollständigen Sistieren der bulimischen Symptomatik. Die Patientin konnte sich durch die vorgegebenen Essensportionen an eine normale Kalorienzufuhr gewöhnen und begann, in einem gemäßigten Ausmaß täglich Ausdauersport zu betreiben. Allerdings kam es kompensatorisch zu einem Abusus von Sorbit haltigen Kaugummis, welche die Patientin auf Grund der laxativen Wirkung missbräuchlich verwendete. Diese Problematik wurde ärztlicherseits thematisiert.

\section{Medikamentöse Behandlung}

Die Patientin wurde auf Fluoxetin eingestellt, welches bei guter Verträglichkeit Leitlinienkonform auf eine Tagesdosis von $60 \mathrm{mg}$ aufdosiert wurde.

Laut Leitlinien der World Federation of Societies of Biological Psychiatry (WFSBP) [13] weisen trizyklische Antidepressiva und Fluoxetin in der Behandlung der Bulimia nervosa ein Evidenzlevel A auf und aufgrund des günstigeren Risikoprofils wird Fluoxetin empfohlen. Die wirksame Dosis liegt mit einer Tagesdosis von $60 \mathrm{mg} / \mathrm{d}$ höher als in der antidepressiven Indikation. Hinsichtlich der körperdysmorphen Störung konnten bei einem systematischen Review der Cochrane Datenbank [10] nur 2 randomisiert kontrollierte Pharmakotherapiestudien gefunden werden. Dabei ergab sich ein positiver Effekt im Vergleich zur Placebobehandlung für Fluoxetin und Clomipramin. Auch bei wahnhaften Formen der körperdysmorphen Störung konnte eine Besserung der Symptomatik durch die Behandlung mit SSRIs erzielt werden, die Kombination mit Antipsychotika ergab keinen signifikanten Vorteil.

\section{Diskussion}

Die dargestellte Fallvignette beschreibt eine 37-jährige Patientin mit einer Bulimia nervosa und einer erstmalig diagnostizierten chronischen körperdysmorphen Störung mit schwerer Ausprägung und wesentlichen Funktionseinschränkungen im beruflichen und sozialen Bereichen. 
Der aktuelle Fall gibt Anlass zur Frage, warum die KDS erst zu einem sehr späten Zeitpunkt diagnostiziert wurde. Die KDS wird insgesamt zu selten als eigenes Störungsbild wahrgenommen und diagnostiziert [7]. Vielleicht wurden körperbezogene Symptome der Patientin im Rahmen einer Körperschemastörung bei einer Essstörung interpretiert. In Subpopulationen mit Essstörungen erfolgten erst in den letzten Jahren Untersuchungen bzgl. des Auftretens von KDS Symptomen. Bei der KDS handelt es sich um eine Körperschemastörung, die sich auf einzelne Körperteile bezieht, bei Essstörungen hingegen ist das Körperschema hinsichtlich der Körperform und -silhouette gestört. Essstörungen sind häufig mit einer Dysmorphophobie vergesellschaftet und beide Erkrankungen weisen ähnliche psychopathologische Charakteristika auf: eine Beschäftigung mit der körperlichen Erscheinung sowie zwanghafte und repetitive Verhaltensweisen (wie z. B. das Überprüfen des Äußeren im Spiegel oder der Körpermaße) [14]. Die Lebenszeitprävalenz einer Essstörung und einer komorbiden körperdysmorphen Störung liegt zwischen 15 bis 32,5\% [14, 15]. In rezenten Untersuchungen wiesen Patientinnen mit einer Bulimia nervosa höhere Komorbiditätsraten für eine körperdysmorphe Störung auf als Patientinnen mit Anorexia nervosa [14, 15]. Essstörungen mit körperdysmorphen Störungen führten zu höheren Hospitalisierungsraten und $\mathrm{zu}$ mehr therapeutischen Interventionen als Essstörungen ohne dysmorphophobe Symptome [14].

Eine andere Erklärung für die späte Diagnose der KDS ist möglicherweise eine fehlende Mitteilung der Symptome seitens der Patientin. Auf Grund der starken Schamgefühle wird die KDS oft als „Schamkrankheit“ bezeichnet. KDS Betroffene sprechen auch bei großem Leidensdruck nur sehr ungern mit Behandlern über ihre Probleme, deshalb sollte explizit danach gefragt werden [7].

Die Patientin schildert den Beginn der Unzufriedenheit mit ihrem Körper im Jugendalter. Aus entwicklungspsychologischer Sicht könnte die lange bestehende Faszination von Barbiepuppen zur Entwicklung des zugrundeliegenden gestörten Körperbildes beigetragen haben. Die „Barbie“ ist Ausdruck plastischer Schönheit mit weit vom durchschnittlichen Frauenkörper abweichenden Maßen. Das Kinderspielzeug beeinflusst das Körperbild von jungen Mädchen [16] bis hin zu Vorstellungen über Genitalien erwachsener Frauen [17]. Normalerweise erfolgt jedoch in der Pubertät und Adoleszenz eine Korrektur der Vorstellungen über einen idealen Körper. Sehr auffällig an unserer Patientin erscheint dabei, dass keine kritische Auseinandersetzung mit den unrealistischen Vorstellungen über den „perfekten Barbie-Körper“ erfolgte, das Schönheitsideal mit den Enttäuschungen über den eigenen Körper bis ins Erwachsenenalter eher rigide beibehalten wurde und entsprechende Abweichungen kosmetischen Korrekturen unterzogen wurden, die jedoch zu keiner anhaltenden Besserung und sogar zu einer Verschiebung der Symptomatik mit Fokussierung anderer Körperteile bei der Patientin führten.

Im Jahr 2015 nahm die Anzahl kosmetischer Eingriffe weltweit insgesamt um mehr als eine Million im Vergleich zum Vorjahr zu [18]. Steigende Zahlen ästhetischer Operationen weltweit geben Hinweise auf eine zugrundeliegende Unzufriedenheit mit dem eigenen Körper in breiten Bevölkerungsschichten. Jedoch scheint die plastische Chirurgie gerade bei der KDS zu keiner Besserung der Symptomatik [9, 19], sondern zu einer iatrogenen Schädigung und Entstellung des Körpers durch vielfache Eingriffe beizutragen. In $\mathrm{Zu}-$ kunft wird es deshalb umso mehr nötig sein, besser auf KDS typische Symptome und zugrundeliegende schwere Selbstwertkonflikte bei Patientinnen und Patienten $\mathrm{zu}$ achten und sie einer entsprechenden psychotherapeutischen und ggf. auch einer psychopharmakologischen Behandlung zuzuführen.

Danksagung Wir bedanken uns herzlich bei der Patientin für die freundliche Zustimmung zur Veröffentlichung ihrer Krankengeschichte. Eine schriftliche Einwilligungserklärung ist vorhanden und kann bei den Autoren angefordert werden.

Funding Open access funding provided by Medical University of Vienna.

Interessenkonflikt M. Gruber, R. Jahn, K. Stolba und M. Ossege geben an, dass kein Interessenkonflikt besteht.

Open Access Dieser Artikel wird unter der Creative Commons Namensnennung 4.0 International Lizenz (http:// creativecommons.org/licenses/by/4.0/deed.de) veröffentlicht, welche die Nutzung, Vervielfältigung, Bearbeitung, Verbreitung und Wiedergabe in jeglichem Medium und Format erlaubt, sofern Sie den/die ursprünglichen Autor(en) und die Quelle ordnungsgemäß nennen, einen Link zur Creative Commons Lizenz beifügen und angeben, ob Änderungen vorgenommen wurden.

\section{Literatur}

1. Grau K, FegertJM, Allroggen M. Körperdysmorphe Störung. Übersichtsarbeit. Z Kinder Jugendpsychiatr Psychother. 2015;43(1):29-35.

2. Veale D, Gledhill LJ, Christodoulou P, Hodsoll J. Body dysmorphic disorder in different settings: a systematic review and estimated weighted prevalence. Body Image. 2016;18:168-86.

3. Driesch G,Burgmer M,HeuftG. KörperdysmorpheStörung. Epidemiologie, klinische Symptomatik, Klassifikation und differentielle Therapieindikation-eine Übersicht. Nervenarzt. 2004;75:917-31.

4. American Psychiatric Association. Diagnostisches und statistisches Manual psychischer Störungen (DSM-5). Göttingen: Hogrefe; 2015.

5. Wegner U, Meisenzahl E, Möller HJ, Kapfhammer HP. Dysmorphophobie - Symptom oder Diagnose. Nervenarzt. 1999;70(3):233-9. doi:10.1007/s001150050427.

6. World Health Organisation. International classification of diseases 10th revision (ICD-10), 2. Aufl. Bern:Huber; 1993. 
7. Stangier U, Hungerbühler R, Meyer A, Wolter M. Diagnostische Erfassung der Körperdysmorphen Störung - eine Pilotstudie. Nervenarzt. 2000;71:876-84.

8. Neumann E. Offener und verdeckter Narzissmus. Paradox eines Konstruktes. Psychotherapeut. 2010;55(1):21-8. doi:10.1007/s00278-009-0714-2.

9. Mester H. Können kosmetische Operationen die ungünstige Weiterentwicklung einer neurotischen Fehlhaltung verhindern? In: Rudolph GEA, TölleR (Hrsg.). Prävention in der Psychiatrie. Heidelberg: Springer; 1984. S. 130-7.

10. Ipser JC, Sander C, Stein DJ. Pharmacotherapy and psychotherapy for body dysmorphic disorder. Cochrane Database Syst Rev.2009; . doi:10.1002/14651858.cd005332.pub2.

11. Küchenhoff J. Der beschädigte Körper und die Angst vor dem Andere: Dysmorphophobie. In: Küchenhoff J (Hrsg.). Körper und Sprache. Theoretische und klinische Beiträge zu einemintersubjektiven Verständnis des Körpererlebens, 2. Aufl.Gießen:Asanger; 2012.

12. Bürgy M. Dysmorphophobie. Das Sich-selbst-fremdwerden als Störung der Kommunikation. Nervenarzt. 1998;69(5):446-50.

13. Aigner M, Treasure J, Kaye W, Kasper S, WFSBP task force on eating disorders. World Federation of Societies of Biological Psychiatry (WFSBP) guidelines for the pharmacological treatment of eating disorders. World J Biol Psychiatry. 2011;12:400-43.
14. Ruffolo JS, Phillips KA, Menard W, Fay C, Weisberg RB. Comorbidityofbody dysmorphic disorder and eating disorders: severity of psychopathology and body image disturbance. Int JEatDisord. 2006;39(1):11-9.

15. Kollei I, Schieber K, de Zwann M, Svitak M, Martin A. Body dysmorphic disorder and nonweight-related body image concerns in individuals with eating disorders. Int J Eat Disord.2013;46(1):52-9.

16. Dittmar H, Halliwell E, Ive S. Does Barbie make girls want to be thin? The effect of experimental exposure to images of dolls on the body image of 5-to 8-year-old girls. DevPsychol. 2006;42(2):283-92.

17. SchickVR, Rima BN, Calabrese SK. Evulvalution: the portrayal of woman's external genitalia and physique across time and current barbie doll ideals. J Sex Res. 2011;48(1):74-81. doi:10.1080/00224490903308404.

18. International Society of Aesthetic Plastic Surgery (ISAPS). Press release - ISAPS statistics for 20152016. https://www.isaps.org/Media/Default/global-statistics/ Global\%20Survey\%20Press\%20Release_V2.pdf. Zugegriffen: 17.07.2017.

19. Bowyer L, Krebs G, Mataix-Cols D, Veale D, Monzani B. A critical review of cosmetic treatment outcomes in body dysmorphic disorder. Body Image. 2016;19:1-8. doi:10.1016/j. bodyim.2016.07.001. 